\title{
NUEVAS FORMULACIONES PARA EL TRATAMIENTO DEL OJO SECO
}

\section{NEW FORMULATIONS FOR DRY EYE TREATMENT}

\author{
VICARIO-DE-LA-TORRE Mํ, HERRERO-VANRELL R ${ }^{1}$, BENÍTEZ-DEL-CASTILLO JM², \\ MOLINA-MARTÍNEZ IT ${ }^{1}$
}

El Síndrome de Ojo Seco (SOS) es una afección ocular, de gran prevalencia, que afecta aproximadamente al $10-15 \%$ de la población. Este síndrome se puede considerar como un conjunto de problemas que surgen a partir de la alteración de la película lagrimal. Dicha alteración tiene como consecuencia inmediata la pérdida de las propiedades ópticas óptimas de la película así como la ausencia de la lubricación, hidratación, protección, nutrición y limpieza de la superficie ocular (1).

El ojo seco, clásicamente, se clasifica en dos grandes grupos: ojo seco acuodeficiente o hiposecretor y ojo seco evaporativo. No obstante dicha clasificación no es buena ya que en el ojo seco de cualquier etiología existen componentes hiposecretores y evaporativos en mayor o menor medida.

Mientras que todos los autores coinciden en el hecho de que el SOS es un resultado de la alteración de la película precorneal, existe una gran controversia en cuanto a la etiología y tratamiento de la enfermedad (2).

Algunos autores plantean el SOS como un problema de base inflamatoria inmune. Su hipótesis estriba en la detección de infiltrados inflamatorios, localizados en glándulas lagrimales y en la conjuntiva, en muestras procedentes de pacientes con SOS asociado y no asociado al síndrome de Sjögren. Otros autores, encaminan sus estudios hacia la demostración de la relación del SOS con un déficit en los niveles circulantes de ciertas hormonas (principalmente andrógenos) basándose, fundamentalmente, en la mayor predisposición de mujeres postmenopaúsicas a desarrollar esta patología (3). La administración de suplementos sistémicos de andrógenos, en estos casos, parece aumentar el volumen de lágrima, aliviando los síntomas del SOS. Parece ser que el efecto beneficioso de estos agentes tiene su origen en el estímulo de la secreción lipídica de las glándulas de Meibomio, mejorando así las condiciones de la película lagrimal, y al efecto antiinflamatorio e inhibidor de la apoptosis lagrimal de los andrógenos.

La superficie ocular se sabe que está formada por el epitelio conjuntival, el epitelio corneal, las glándulas lacrimales accesorias y las glándulas de Meibomio. Dicha superficie se encuentra recubierta por una película continua, denominada película precorneal o film lagrimal. Hasta hace algunos años, la estructuración teórica, generalmente aceptada, incluía tres tipos de componentes (lipídico, seroacuoso y mucinoso) repartidos en tres capas: lipídica, acuosa y mucinosa. Estudios recientes consideran una estructura formada por los componentes acuoso-proteicos y mucinosos combinados para formar un gel hidratado. A su vez, este gel quedaría protegido por una película de carácter lipídico, cuyos componentes serían producidos principalmente por las glándulas de Meibomio y cuya función sería impedir la evaporación de la lágrima y mejorar la estabilidad de la película lacrimal. Esta película al encontrarse alterada en el ojo seco condiciona la elección de los vehículos empleados en la fabricación de las formulaciones.

Independientemente del origen de la enfermedad, la terapia del SOS se centra, fundamentalmente, en mejorar los síntomas de la enfermedad (4). Dentro de los tratamientos se incluyen: sustitución de la lágrima natural (por medio de lágrimas o saliva artificiales), preservación de la lágrima existente (mediante oclusión del sistema de drenaje o por

\footnotetext{
1 Departamento de Farmacia y Tecnología Farmacéutica. Facultad de Farmacia. Universidad Complutense. Madrid. España.

2 Departamento de Oftalmología. Hospital Universitario San Carlos. Madrid. España.

E-mails: marta_vicario@yahoo.es / rociohv@farm.ucm.es / iremm@farm.ucm.es / benitezcastillo@terra.es
} 
medios que disminuyan su evaporación) o estimulando una mayor producción de lágrima natural (a través de fármacos secretagogos o lacrimomiméticos). Actualmente el tratamiento con lágrimas artificiales constituye casi un $80 \%$ de la terapia. Las formulaciones más novedosas en el mercado constituyen diferentes tipos de preparados: soluciones hipotónicas, lágrimas que contienen lípidos para prevenir la evaporación del agua de la película lagrimal, lágrimas con propiedades bioadhesivas para incrementar la retención de agua y formulaciones que contengan sustancias protectoras del estrés celular provocado por la hipertonicidad de la lágrima.

En cualquier caso: ¿Cuál sería la formulación ideal? Para nosotros sería aquella diseñada a partir de la estructura de la superficie ocular y del conocimiento de la función de cada uno de los integrantes de la misma. Probablemente resultaría una conjugación de las formulaciones existentes en el mercado.

La lágrima artificial ideal debería ser hipotónica (hasta un valor de tonicidad adecuado y sin que produjera reacciones adversas) y contener lípidos semejantes a los de la película lagrimal. Se sabe que dichos lípidos son de naturaleza polar (fosfolípidos, esfingomielina, ceramidas y cerebrósidos) y no polar (ésteres de cera, ésteres de colesterol, triglicéridos, ácidos grasos libres e hidrocarbonos). Además, se deberían incluir componentes bioadhesivos no sólo capaces de incrementar la retención de agua sino con el fin de aumentar el tiempo de contacto con la superficie ocular (los polímeros bioadhesivos son capaces de interaccionar con los ácidos siálicos de la mucina) (5) y por último incorporar sustancias activas protectoras del epitelio córneal. Si además, se pudieran incorporar en dichas formulaciones hormonas con actividad androgénica o bien sustancias activas capaces de aumentar la producción de lágrimas o reducir la inflamación sería de gran interés.

En conclusión, tenemos ante nosotros un panorama excitante, el de una enfermedad de nuestro tiempo en constante crecimiento y ante la que las nuevas terapias precisarán de la investigación multidisciplinar basada en la tecnología farmaceútica y en la oftalmología básica y aplicada.

\section{BIBLIOGRAFÍA}

1. Baudouin C. Dry eye: an unexpected inflamatory disease. Arch Soc Esp Oftalmol 2001; 76: 205-206.

2. Pflugfelder SC, Solomon A, Stern ME. The diagnosis and management of dry eye: a twenty-five-year review. Cornea 2000; 19: 644-649.

3. Sullivan DA, Krenzer KL, Sullivan BD, Toll DB, Toda I, Dana MR. Does androgen insufficiency cause lacrimal gland inflammation and aqueous tear deficiency? Invest Ophthalmol Vis Sci 1999; 40: 1261-1265.

4. Calonge M. The treatment of dry eye. Surv Ophthalmol 2001; 45: S227-S239.

5. Herrero-Vanrell R, Fernandez-Carballido A, Frutos G, Cadorniga $R$. Enhancement of the mydriatic response to tropicamide by bioadhesive polymers. J Ocul Pharmacol Ther 2000; 16: 419-428. 